\title{
Presidential Lecture \\ Changing Features of Diseases and Their Management
}

\author{
Yasushi UedA**, MD
}

\section{PREFACE}

Recently it has been stated that there are remarkable alterations in the nature of diseases in every field of medicine, and this is no exception in internal medicine. It is considered a matter of consequence to comprehend the fact and to manage for proper diagnosis, prognosis and treatment. Such a changing trend of diseases owes much to the progress in modern medicine, namely progress in analytical methods and treatment for the quality of diseases, but on the other hand those influences as changes in social architecture, enviroment and economical state are not to be neglected. In this country of particular such rapid changes of social as well as economical background to influence and to enhance the trend of changing nature of diseases have taken place.

\section{[A] The fact and features of changing trends of diseases}

Many factors are considered present for above trends but it may be stated that principal causes are due to progress in basic medicine, analytical method for epidemio$\log y$, diagnosis and therapy in clinical medicine.

(1) Decrease and disappearance of certain diseases

First to be pointed out for remarkable decrease and disappearance of diseases are infectious diseases. Of the many instances small pox may be a representative one. Ekiri and lymphgranuloma venereum have virtually disappeared in this country. In Japan tuberculosis and typhoid fever are gradually to fade away owing to potent antibiotics available and also to improvement of social enviroments such as diet and housing condition. Such decrement or fading of infectious diseases clearly owes to the progress in preventive medicine and modern therapy but also to the changes in social enviroment observed in this country. (2) Alteration of disease entity

Naturally disease entities are changing in accord with progress in medicine and the fact lies in the category of changing feature of diseases. There are many those instances but here glomerulonephritis may be chosen for a represntative example. Since first described by R. Bright in 1827 for its entity, 50 years had elapsed before the name glomerulonephritis appeared in the literature in 1876. Nextly Ellis' clinical classification was introduced in 1942 then followed by that of renal biopsy in 1960 which eventually resulted in further refinement for the classification today. Present classification of course will be subjected to more refinement in accord with new research and diagnostic technology and thus approaches to true nature of the disease.

(3) Progress in methodology of diagnosis

'Through many years' clinical research and pathological correlations entities of

\footnotetext{
*Presented at the 76 th Annual Meeting of the Japanese Society of Internal Medicine, April 6, 1979, in Tokyo, Japan.

**Prof of The Second Department of Internal Medicine, The Jikei University School of eMdicine, Tokyo.
} 


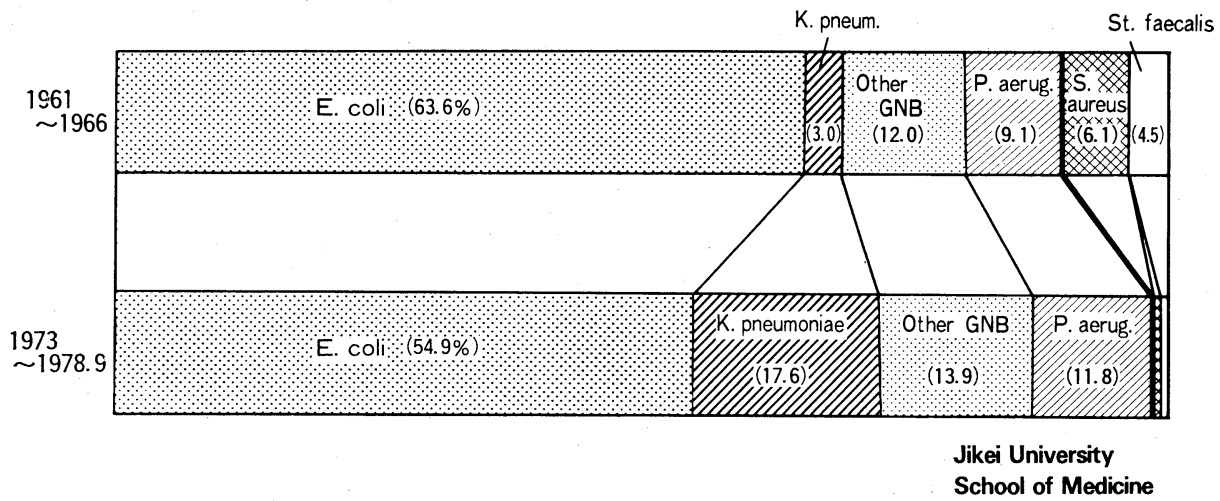

Fig. 1. Changes of Causative Organisms for Ghronic Pyelonephritis.

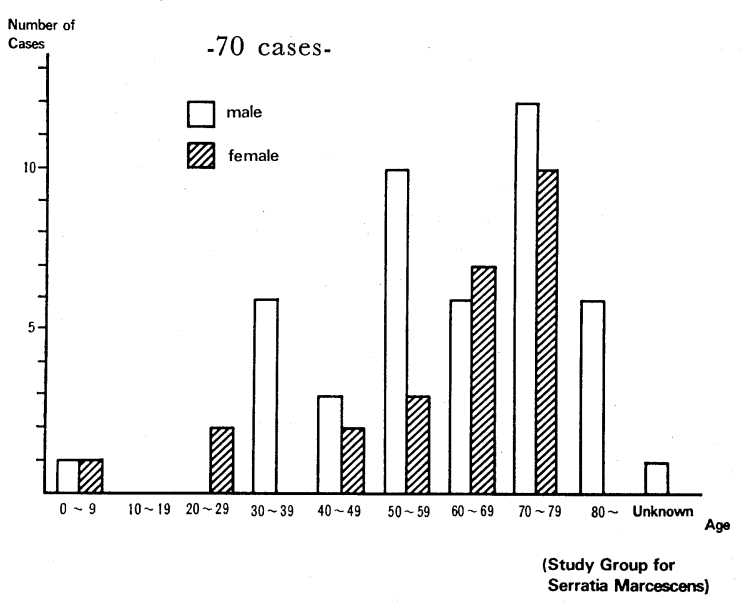

Fig. 2. Sex and Age Distribution os Serratia Infections in the Field of Internal Medicine.

certain diseases seems to be fairly settled but as yet these may be entirely changed due to recent developments of new diagnostic apparatus. For instance application of image reconstructive apparatus such as $\mathrm{CT}$ and echo-ultrasound tomography are to be raised. CT scan particularly was first applied to brain now extending toward whole body to yield an excellent diagnostic tool in clinical medicine.

(4) Progress in therapeutic medicine

While progress in therapeutic medicine has much contributed to the treatment of various diseases but at times has acted to change the disease entirely to the point where the disease was refractory to therapy itself. In infectious disease for instance there have been reported many of literatures for changing feature of diseases. The fact is closely related to changes of species of pathogenic organisms. For these changes introduction of new potent drugs, or its abuse and also increased multiplicity of host factors are being considered.

Fig. 1. denotes such a changing feature of causative organisms for chronic pyelonephritis and also in Fig. 2. a present status of increasing incidence of Serratic marcescence in elderly populations are illustated. There are problems of opportunistic infections and hospital infections for changing features of infectious diseases. These two play a major role for it but some are related to progress in modern medicine and also to changes in aged population recently observed. And further it is intermingled with increased incidence of drug resistant organisms due to abuse of drugs.

(5) Appearance of new diseases

It has been well known that new diseases are ever discovered in every field of medicine. On the other hand there are many diseases in which a long survival of patients has been made possible contrary to hitherto concept of medicine. Some of these may be agreed upon as new diseases 


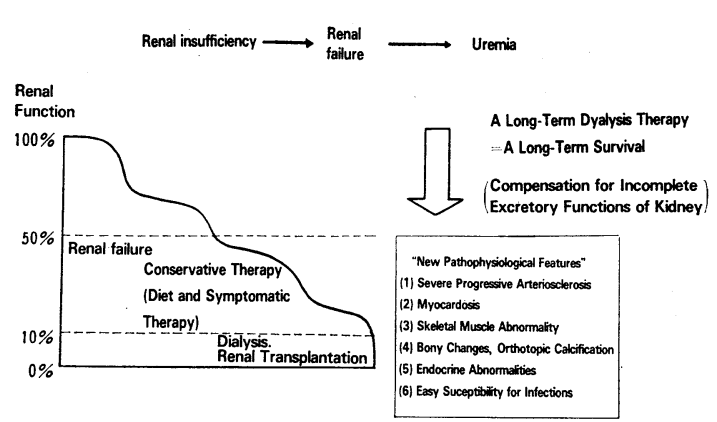

Fig. 3. Changes of Pathological Features in Chronic Dialysis.

in view of new pathophysiology. For these instances included are patients with chronic renal failure under regular intermittent dialysis and receipients of renal transplantation. These patients actually possess new pathophysiology and in authors opinion this is new kinds of renal diseases. Since these patients possess specific pathophysiology, totally different approach from previous concept is mandatory for medical and social management of the diseases. In Fig. 3. appearance of new pathological features in patients with chronic renal failure under regular dialysis is shown. It is self-explainatory that one would survive for a longer period even with a presence of several abnormal state such as bony abnormalities, vascualr changes and endocrine disturbances. It is not appropriate to observe these simply as prolongation of life but one should recognize them as new diseases with irrversible pathophysiology and hence should cope with new management.

(6) Increase of elderly populations

From 1980 th toward twenty-first century one of the characteritic changes is expected to be a rapid increase of popuation and prime reason is considered to be an increase of elderly population. The fact consequently are to result in higher incidence of diseases and its pathophysiology will be more varied as compared with those of younger adults. Furthermore changing trend of diseases in the aged appears to be more rapid with graver prognosis.

As the trend of increasing elderly population is ever present in this country one sholud pay a special attention to it in view of changing feature of diseases.

Population survey in this country shows that increase indice in 1975 as compared with that of 1935 are two folds at the age of 50 th, 2.4 folds at 60 th, 3 folds at 70 th and 3.5 folds at 80 th. This trend is expected to be accelerated in 1980 th. It has been documented that disease rates has increased 2.5 folds in the past 25 years, and the fact derives mainly from an increase of elderly populations. Those ischemic heart diseases, cerebrovascular diseases, hypertension and diabetes mellitus naturally increase with age but in the aged the features of diseases have been more varied as compared with those of younger adults. When complicated with other diseases for example infectious diseases, the underlying diseases accelerate the changing features.

\section{[B] Management for Changing Feature of Diseases}

Changing feature of diseases hitherto stated is thought to continue and some would accelerate the rate of changes. Progress in therapy is one of the principal causes for the change of diseases but on the other hand it is also important for the management. As a mean of management for changing feature of diseases there are a problem of developing new drugs. Many difference of opinions are present on this problem such as idea of newly developement, evaluations for effects and safety, and untoward reaction but all are left open for further eradications. It is to be emphasized that new ideas from doctors as the user of drugs should first be brought up and on top of this newly developement and production of drugs are to be made which potentially enable to cope with changing feature of diseases.

Surrounding the factors for changing features of diseases as represented by fixed state of increased aged population, there 
are changes in social as well as economical enviroments and medicare system proper to this country.

On the other hand to be raised are the influence of recent trend in world-wide abnormal climate, specific living enviroments in large cities, differences of living standards (state of living enviroment) between cities and remote area or isolate islands. It may be stated that all of or part of above factors are closely related to the changing feature of diseases today and act as the causative factors to accelerate the trend. These enviromental factors would be expected to increase year by year and never to decrease therefore close observation not only from the side of medicine but also from the side of enviromental factors are necessary for the management of diseases. But one faces a dilenma in the management from enviromental factors around the diseases as there are questions for active measures and difficulties for actual practice. Pollution problem is a good example for it.
It may be consented that the changing feature of diseases in view of non-medical aspect is defined as a kind of disease of civilization derived from progress in human culture. But none the less it is not to be left open.

\section{CONCLUDING REMARKS}

The fact and proper measures for changing features of diseases have been briefly discussed mainly from the aspect of internal medicine. For Japanese medicine ever to step foward to twenty-first century comprehension of these problems are of the prime importance. A recent survey in Japan indicates that there are increased trend of medicare rate in cardiovascular and metabolic diseases while decreased rate of respiratory, contagious and parasite diseases. These figures may be a key reference for prospective views of changing features of diseases. Many of the diseases are expected to enhance the rate of changes and the very fact shall not be neglected for considering better medicine tomorrow. 